\title{
Galactic flows and the formation of stellar clusters
}

\author{
Romas Smilgys ${ }^{1}$ and Ian A. Bonnell ${ }^{1}$ \\ ${ }^{1}$ Scottish Universities Physics Alliance (SUPA), School of Physics and Astronomy, University \\ of St. Andrews, North Haugh, St. Andrews, Fife KY16 9SS, UK \\ email: rs202@st-andrews.ac.uk
}

\begin{abstract}
We investigate the formation of stellar clusters from a Galactic scale SPH simulation. The simulation traces star formation over a 5.6 Myr timescale, with local gravitational instabilities resulting in $\sim 10^{5}$ solar masses of star formation in the form of sink particles. We investigate the time evolution of the physical properties of the forming clusters including their half-mass radii, their energies and the depletion time of the gas. Star formation is driven by the large scale flows which compress the gas to higher densities where self gravity takes over and collapse occurs. We show that the more massive clusters (up to $\sim 2 \times 10^{4}$ solar masses) gather their material from of order $10 \mathrm{pc}$ due to these large scale motions associated with the spiral arm passage and shock. The bulk of the gas becomes gravitationally bound near 1-2 Myr before sink formation, and in the absence of feedback, significant accretion ongoing on longer timescales. We trace the hierarchical merging process of cluster formation which naturally results in age spreads of order the crossing time of the original region which provides the gas reservoir for the cluster.
\end{abstract}

Keywords. stars: formation, ISM: clouds, (Galaxy:) open clusters and associations: general

\section{Introduction}

Star formation is one of the key processes in understanding galaxies evolution and physical conditions how gas are being transformed into stars. Most stars are believed to be formed in clusters (Lada \& Lada (2003), Gutermuth et al. (2009), Bressert et al. (2010)) such that establishing the physical conditions for the formation of stellar clusters is crucial in order to develop a comprehensive theory of star formation. However, physical conditions of cluster formation are not well understood.

Observations of recent years are starting to unveil much more details (i.e. Peretto et al. (2013)), but they alone provides only a single snapshot in cluster evolution. This is where numerical simulations comes into their role by providing insight into the physical process of cluster formation. However, most of cluster formation based simulations (Bate et al. (2003), Bonnell et al. (2003)) used idealised initial conditions, ignoring how such initial conditions arise, or even if they are realistic. So large scale Galactic dynamics are getting ignored in these simulations.

First attempts to study star formation including Galactic scale flows were made by Bonnell et al. (2006). Bonnell et al. (2013) produced a set of 3 nested simulations (Global disc simulation, high resolution Cloud re-simulation and Gravity re-simulation) to cover wide range of scales. Global disc simulation used entire Galactic disc. Cloud simulation used high density region from Global simulation within about $2 \mathrm{kpc}$ size. And finally Gravity simulation used 250 pc region from Cloud simulation. These simulations shown that there is possible to study star formation on cloud scale without excluding galactic spiral arm passage through ISM. We continue to investigate origin of star formation 


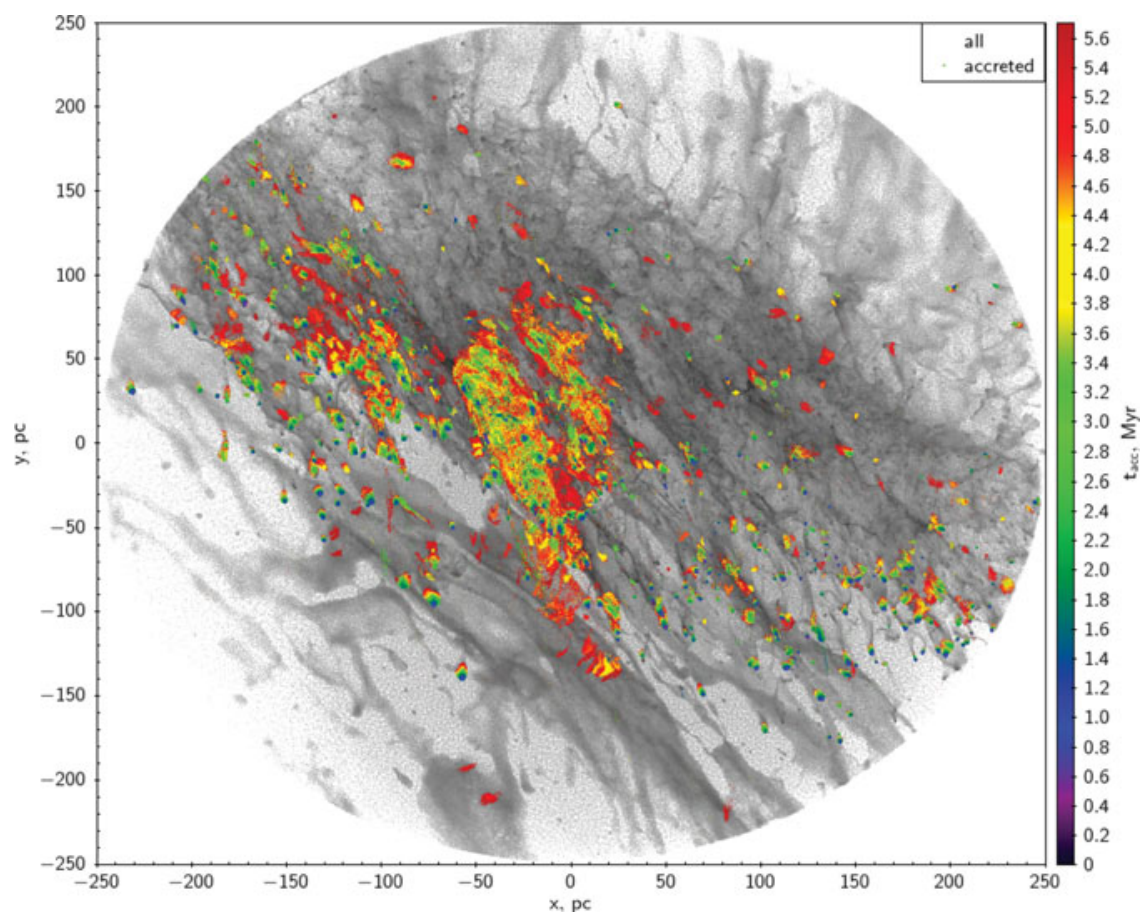

Figure 1. Initial conditions of Bonnell et al. (2013) simulation in terms of $\mathrm{x}-\mathrm{y}$ positions. Grey colour shows where are all gas particles located, while coloured ones show where accreted particles (We denote the gas particles which are involved in star formation as accreted particles) are located. Accreted particles are colour coded by accretion time, which shows when they will be accreted.

between hundreds of parsecs scales down to sub-parsec scales and the formation of stellar clusters. Lagrangian nature of SPH simulations allows easily to follow and recover evolution of cluster forming regions, and to seek where, when and how star formation is triggered.

\section{Galactic flows}

We start with Bonnell et al. (2013) Gravity simulation initial conditions. These conditions were inherited from a set of nested simulations based on larger galactic and spiral arm scales, setting the initial positions and velocities of the SPH particles. We plot all the particles of the Gravity simulation in Figure 1 in greyscale.

The Lagrangian nature of particles in SPH allows us to follow positions, velocities and other parameters over the time. We can thus follow the star formation process, the onset of self-gravity, collapse and accretion over all time steps. We select all these accreted gas particles and plot on the top of all simulation gas particles, and colour coding them by accretion time (Figure 1).

The gas particles in Figure 1 clearly show the presence of the spiral arm, with gas compressed as it exits the spiral arm. Darker grey areas show larger density regions. Gas particles that are subsequently involved in star formation are initially located in the densest parts of the simulation. In the middle is visible a large chunk of accreted gas extending $\sim 150 \mathrm{pc}$ from upper to lower side. This is the main star forming region, where 10 massive clusters form in 5.6 Myr. 


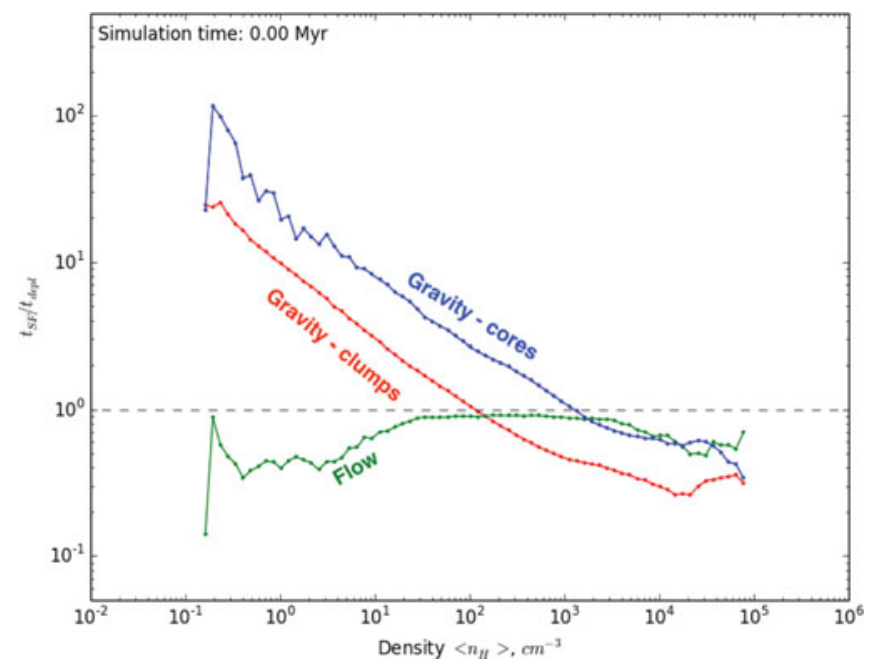

Figure 2. Star formation and depletion time ratio vs gas SPH density diagram at the start of simulation. Star formation can be explained only when this ratio is lower than 1 . The diagram shows that at large scales (low densities) star formation is driven by Galactic flows, while on small gravity takes over.

Colours of accreted particles show that early star formation (blue) is concentrated into tiny clumps, which collapse in $\sim 1$ Myr to form first sinks. These clumps are surrounded by green, yellow and red regions, which are widespread over much larger volumes. This indicates that the gas, which forms stars later, is still more far away from star formation locations than the gas forming stars earlier. So early star formation is likely to be driven by the rapid collapse of local dense regions.

In order to understand what is driving star formation, we compare the actual star formation timescale, the depletion time, against simple predictions for the timescale for star star formation. These include the free fall time of the region depending on the local gas density, the collapse time of the individual star/sink, and the timescale for compression due to the large scale kinematics of the gas flows. We use predicted star formation and depletion time ratio (Figure 2) as a function of gas density to understand star formation triggering mechanisms over such large range of scales. All three predicted star formation times can be expressed in a ratio with depletion time, which is real time in the simulation required for particles to reach the location where they are accreted by a sink, or formed into a new one. The predicted timescale must be less than the actual (depletion) timescale as recorded by the simulation if the associated physics is driving the star formation.

Figure 2 show that gravity drives star formation only at larger densities, while only flow can explain star formation of low densities. In contrast, at lower densities it is the large scale flows from the Galactic shock which drives compression until larger densities are reached where self-gravity can take over.

\section{Stellar clusters}

Soon after looking at the positions of sinks at the end of the simulation we noticed that sinks tend to be not alone but often forms tight groups (clusters of sinks). These clusters can be used as references for massive star clusters. We wrote an algorithm to detect these clusters on every time step of the simulation. The detection is based on a 


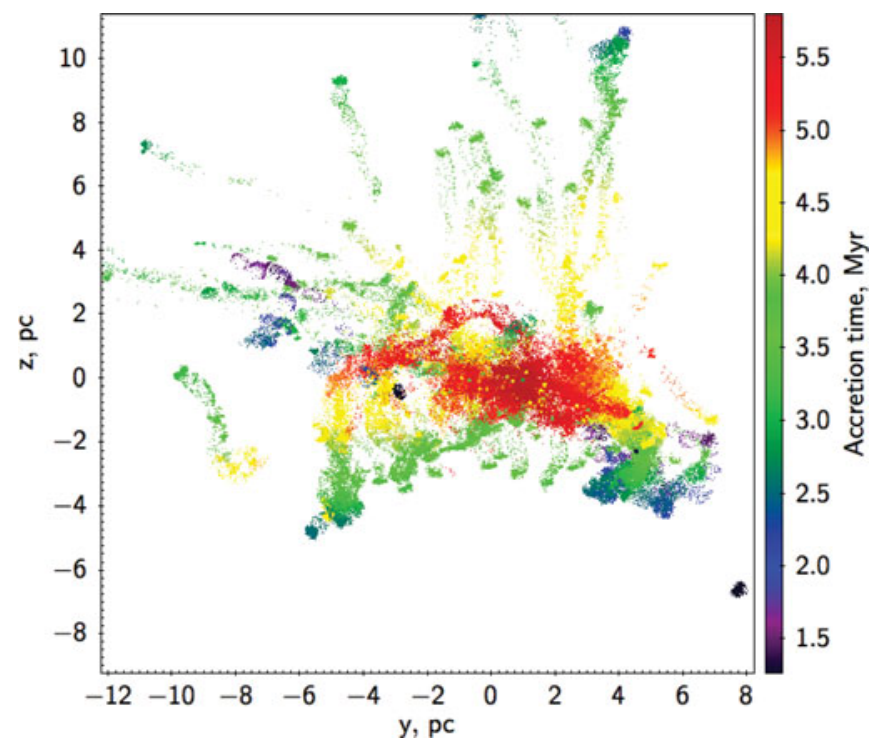

Figure 3. Star formation traces inside most massive cluster in terms of $y-z$ positions. Colours represents when star formation is happening. Diagram shows that early star formation takes place in smaller groups of clumps, which later assemble into final larger cluster.

cluster definition of at least 6 gravitationally bound sinks where members are in at least 1 pc neighbourhood with other cluster members. As a particular case we look at final simulation clusters and trace their formation history backwards in time.

Firstly we look at all sink formation and accretion events in a position-position diagram (Figure 3) of the most massive final cluster. We can trace star formation history and hence how the cluster formed and grew. The final positions of each particle is plotted relative to the cluster centre of mass. The colour represents the time at which accretion occurred. We can clearly see there paths of ongoing accretion pointing towards the centre of cluster. These paths are left by moving sinks, which keeps accreting gas along their way. The early star formation is quite isolated, where the first sinks form (blue colour). At later times sinks are migrating towards the cluster centre while accreting gas along their paths (green and yellow tracks) and finally form 2-3 pc size cluster in the middle. There are also visible sub-grouping in the early to mid star formation, which indicates that sub-clusters are starting to form earlier, and later merge to form larger cluster.

We repeat our cluster finding procedure over the entire field to find also smaller and less massive clusters. Over 10 clusters with masses $>1000 M_{\odot}$ form in the main star forming region with sizes of 1-2 pc and distances between clusters of $\sim 10 \mathrm{pc}$. To show the variety of clusters we plot their mass-radius relation (Figure 4), where we use half-mass radii to express cluster sizes. We plot the evolution of mass-radius relation for all time steps. The diagram shows a clear trend that more massive clusters are also larger in size. The earliest clusters form at $\sim 2$ Myr from the start of the simulation with masses up to 1000 - $2000 M_{\odot}$. At later times, clusters grow most significantly in their masses while only slightly increasing their sizes (at $\sim 4$ Myr we see most massive clusters of $\sim 5000 M_{\odot}$ and at the end of simulation reaching almost $\left.30000 M_{\odot}\right)$.

Finally we look at the question of stellar age spreads within clusters. We reconsider star formation traces seen in Figure 3 and plot accretion time versus distance from the cluster mass centre where accretion occurs (Figure 5). Tracks are shown for 3 most massive clusters and coloured by cluster to which they belong. The evolutionary tracks move 


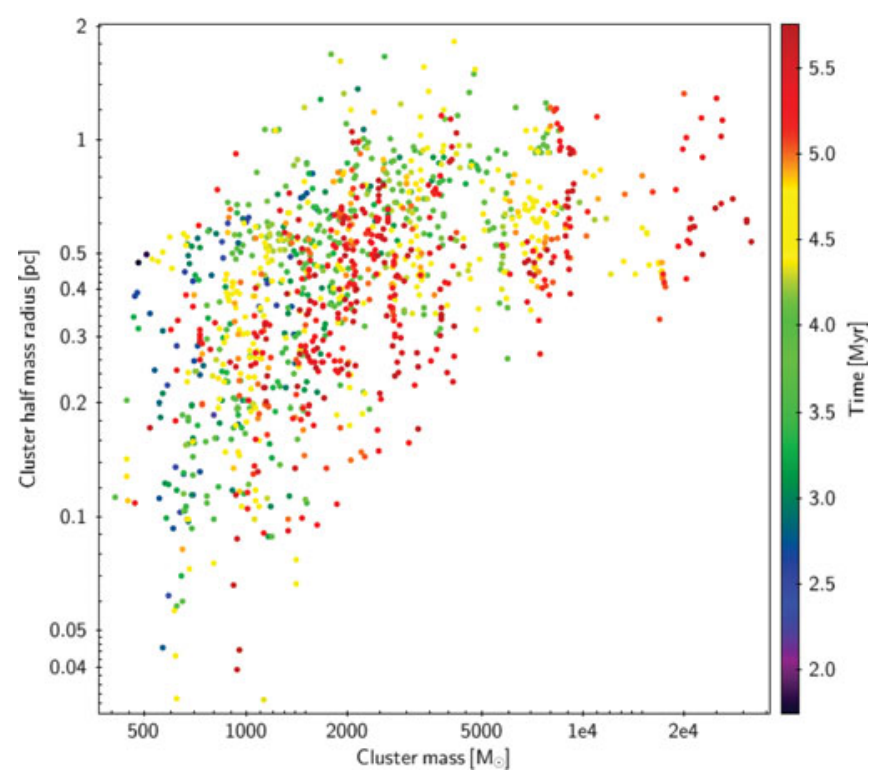

Figure 4. Evolution of mass-radius relation for simulation clusters. Diagram shows that more massive clusters are also larger in size and when time passes clusters grow mostly in mass, while keeping similar sizes.

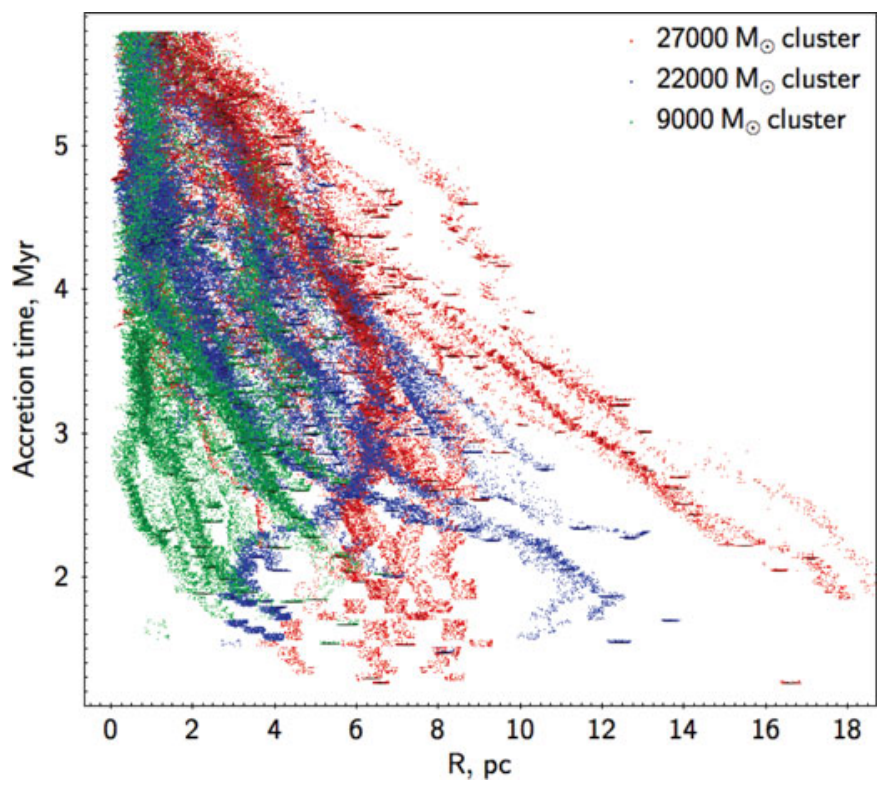

Figure 5. Accretion history of 3 most massive final cluster. Diagram shows that accretion inside clusters is happening continuously over several Myr along sink paths towards cluster centre. This indicates possible age spreads inside cluster, which assembles from smaller clusters.

towards smaller radii over all times due to the hierarchical nature of the fragmentation and subsequent merging. The tracks start at $1.5-2 \mathrm{Myr}$, which is the start of the formation of individual sinks. Sink formation is denoted as strong $0.5 \mathrm{pc}$ wide horizontal bars at the beginning of the tracks. Clusters keep accreting gas all the time up to the 
end of simulation. However, cluster merging from several sub-clusters indicates potential stellar age spreads up to several Myr.

\section{Summary}

We analysed data produced by the SPH simulation, which uses Galactic dynamics to see how star formation is triggered and how do stellar clusters form. We found that gravitationally unstable clouds start to collapse and first stars and clusters after 1-2 Myr. The local collapse is followed by the larger region global collapse, creating more massive clusters. Galactic flows are responsible for compressing these clouds and explains star formation from large scales. We found that forming clusters starts to form widespread hierarchal merging make place to form larger clusters. More massive clusters are also larger in size and mass-radius relation show that forming clusters are mostly increasing in mass while sustaining similar sizes over the time. Cluster merging show potential age spread of several Myr.

\section{References}

Bate M. R., Bonnell I. A., Bromm V., 2003, MNRAS, 339, 577

Bonnell I. A., Bate M. R., Vine S. G, 2003, MNRAS, 343, 413

Bonnell I. A., Dobbs C. L., Robitaille T. P., Pringle J. E., 2006, MNRAS, 365, 37

Bonnell I. A., Dobbs C. L., Smith R. J., 2013, MNRAS, 430, 1790

Bressert, E., Bastian, N., \& Gutermuth, R., et al., 2010, MNRAS, 409, L54

Gutermuth, R. A., Megeath, S. T., \& Myers, P. C., et al., 2009, ApJS, 184, 18

Lada, C. J., \& Lada, E. A., 2003, ARAA, 41, 57

Peretto N., Fuller G. A., Duarte-Cabral A., Avison A., et al., 2013, A\&\&A, 555, A112 